\title{
The Relationship Between Mindfulness, Cognitive Intrusions, and Recollection: An ERP study
}

\author{
Tomasz Jankowski and Paweł Stróżak
}

Institute of Psychology, the John Paul II Catholic University of Lublin, Poland

ABSTRACT

The purpose of the present study was to investigate whether mindfulness and cognitive intrusions predict recollection. Using electroencephalogram methodology, we investigated 31 participants who performed a recognition task and reported situational mindfulness as well as task-irrelevant (TII) and task-relevant intrusions (TRI). We used behavioral measures (response accuracy) to analyze performance effectiveness and event-related potentials (ERP) to measure processing efficiency (compensatory processes) associated with performance of the task. Results suggest that being mindful during a task slightly improves recognition of old and new but not similar probes. Although worrying about the outcomes (i.e., TRI) facilitates improvement in true recognition of old probes, it also impairs correct rejection of new probes. Moreover, TRI predicted the strength of ERP effects associated with compensatory processes involved in recollection. We conclude that mindfulness slightly improves recognition without involvement of the compensatory effort and worrying partly increases responding accuracy at the cost of diminished processing efficiency.

\section{INTRODUCTION}

Dual-process models of recognition distinguish two independent mechanisms that guide our memory judgments: a vague, yet conscious feeling of familiarity, unsubstantiated by any specific characteristics of the past; and recollection of detailed, contextualized information about the previous events (Yonelinas, 2002). A simple example can illustrate the distinction between these two processes. Let us imagine that we chance upon a person who looks like someone we met before but we cannot recall the context of the previous meeting. At this moment, we would probably experience a strong familiarity effect, indicating that we know the person. However, familiarity does not provide any further information that allows for identifying the source of this experience. If we searched our memory, we could retrieve more detailed and con-

Corresponding author: Tomasz Jankowski, Institute of Psychology, The John Paul II Catholic University of Lublin, Al. Racławickie 14, 20-950 Lublin, Poland. E-mail: tojankowski@kul.lublin.ok 
textualized information sufficient to recall the source of the feeling of knowing the person.

\section{Mindfulness and Episodic Memory}

Recollection is not always successful because it requires intentional and conscious processing of limited information-cognitive resources. In line with this claim, several studies found that recollection is strongly related both to attentional resources (e.g., Knott \& Dewhurst, 2007; Rosenstreich \& Goshen-Gottstein, 2015) and to working memory capacity (WMC; e.g., Elward, Evans, \& Wilding, 2013). It seems reasonable to expect that factors enhancing the availability of attentional resources and WMC will also improve retrieving information from episodic memory. The natural candidate for such a variable is mindfulness (Jankowski \& Holas, 2014; Levi \& Rosenstreich, 2018), that is, a state of mind that "emerges through paying attention on purpose, in the present moment, and nonjudgementally to the unfolding of experience moment by moment" (Kabat-Zinn, 2003, p.145). Although mindfulness is often associated with meditation practice, it can be also conceptualized as receptive and open attention and awareness directed toward ongoing events; as such, it is a natural and spontaneous state of mind that fluctuates in time and can be experienced with no meditational practice (Brown \& Ryan, 2003).

Several studies showed that mindfulness is associated with improvement in cognitive functions (van Vugt, 2015), particularly with the improved allocation of attentional resources (e.g., Norris, Creem, Hendler, \& Kobe, 2018) and increased WMC (e.g., Jha, Krompinger, \& Baime, 2007). Mindfulness was also found to predict more specific autobiographical memories, which suggests its positive association with improved recollection (e.g., Heeren, van Broeck, \& Philippot, 2009). Brown, Goodman, Ryan, and Anālayo (2016) found significant correlations between state mindfulness and improved recognition in the remember/know task. Similarly, Lloyd, Szani, Rubenstein, Colgary, and Pereira-Pasarin (2016) showed that a brief mindfulness exercise induced immediately before retrieval (but not encoding) decreased the false alarm rate in a recognition task (without affecting the hit rate).

Despite the results mentioned above, the relationship between mindfulness and episodic memory is far from being clear. Alberts, Otgaar, and Kalagi (2017), for example, found no evidence that the brief mindfulness exercise enhances memory performance. In an earlier study, Alberts and Thewissen (2011) found that mindfulness did not increase overall memory performance, but reduced recall of negative words. Moreover, several studies showed an adverse effect of mindfulness on memory, suggesting that mindfulness increases false memories and incorrect memory recall (e.g., Wilson, Mickes, StolarzFantino, Evrard, \& Fantino, 2015).

Levi and Rosenstreich (2018) have made a comprehensive review of findings and tried to explain the inconsistency in results on the relationship between mindfulness and episodic memory. They proposed a working model connecting three facets of mindfulness with different systems of memory. According to this model, only one facet of mindfulness, that is, acting with awareness, is hypothesized to influence recollection directly. Levi and Rosenstreich also proposed that mindfulness affects the processes of scanning and evaluating memory but not memory itself. They emphasized a need for further research on mindfulness and episodic memory.

In this context, the first aim of the present study was to verify Levi and Rosenstreich's (2018) suggestions and examine the relationship between mindfulness and recollection processes. We focused on acting with awareness (Brown \& Ryan, 2003) as the mindfulness facet particularly important for recollection. Because previous studies found effects of dispositional mindfulness only on familiarity, but not on recollection (Rosenstreich \& Ruderman, 2016; 2017), and state mindfulness was associated with remembering accuracy in at least one study (Brown et al., 2016), we decided to investigate the latter.

\section{Cognitive Intrusions and Episodic Memory}

Some authors criticize investigations of mindfulness that use selfreport measures instead of inducing state mindfulness via meditation practice (e.g., Grossman, 2011). They argue that mindfulness defined and measured as acting with awareness does not differ from other similar phenomena such as lapses in attention or mind-wandering. This criticism should be taken seriously and tested empirically, particularly in cognitive tasks, because full attention allocation to the task requirements (e.g., recalling detailed information) can be reduced due to cognitive intrusions that automatically arise in the participants' minds. Therefore, in the present study we tested the relationship between mindfulness and recollection under control of cognitive intrusions.

Task-irrelevant intrusions (TII) are an example of mind-wandering during a task. These usually capture one's attention and overload the WMC (Kane et al., 2007). In the case of a recognition task, this may lead to impaired recollecting of contextual information. Several studies have already confirmed this expectation: Maillet and Rajah (2013) found, for example, that mind-wandering negatively impacts memory retrieval. Thomson, Smilek, and Besner (2014) showed that this effect is particularly pronounced when comparing young and older participants, as well as under prior semantic encoding instructions compared to perceptual encoding instructions.

Task-relevant intrusions (TRI) are the second type of thoughts that can be experienced by a person during a recognition task. While TII occur in situations where the content of awareness is decoupled from the task, TRI concern situations when attention is directed toward task reappraisal (Smallwood et al., 2004). In other words, TRI occur when information about task performance is processed offline; they represent self-evaluative thoughts (i.e., worries) which-according to cognitive interference theory (Sarason, Sararon, Keefe, Hayes, \& Shearin, 1986) - are triggered by a performance-based anxiety or/and uncertainty. Thus, as an additional process using attentional resources, TRI interfere with online processing of information about a task.

However, in contrast to TII, TRI might have ambiguous effects on task performance. On the one hand, worrying overloads the attention resources at the early stage of recollection (Hayes, Hirsch, \& Mathews, 2008). On the other, in high-demanding tasks, TRI can trigger compensatory strategies (e.g., effortful processing or increasing use of 
processing resources) at a later stage of processing to achieve task goals (Eysenck, Derakshan, Santos, \& Calvo, 2007; Eysenck \& Derakshan, 2011). Thus, while TII are expected to reduce the correct response rate, TRI are expected to increase the effort put into recollection. In terms of Eysenck's theory (Eysenck et al., 2007) TII impair performance effectiveness (i.e., accuracy rate) and TRI affect processing efficiency (i.e., amount of effort required to respond correctly).

We found no studies that investigated the relationship between mindfulness and recollection (measured both in terms of performance effectiveness and processing efficiency) in the context of cognitive intrusions. Thus, the second aim of the present study was to dissociate mindfulness effects from mind-wandering effects in a recollection task. We focused on two problems that-according to our best knowledge-have not been investigated up to now. The first refers to the relationship between mindfulness, cognitive intrusions, and performance effectiveness (accuracy rate). Contrary to Grossman (2011) but in line with Brown, Ryan, Loverich, Biegel, and West (2011), we distinguish between acting with awareness and mind-wandering. However, we agree that these phenomena are correlated. Therefore, because mindwandering undermines attentional control, we expected that TII reduce mindfulness during a task and hence diminish the retrieval of contextual information.

The second problem concerns the relationship between mindfulness, cognitive intrusions, and performance efficiency (i.e., effort needed to perform a task correctly). Based on Eysenck and Darkshan's (2011) theory, we assume that detailed information may be retrieved from memory in two stages. In the first stage, easily accessed, contextual information stored in memory is retrieved. If the quality of information is high, the signal about the proper response is triggered. However, if the signal is weak due to uncertainty or impoverished information, compensatory processes such as additional source monitoring are activated. Because mindfulness is related to improved attentional control, it is hypothesized to positively impact the retrieval of contextual information in the first stage. In other words, because high mindfulness leads to less distraction in the first stage, there is less need to compensate for attentional disturbance in the second stage. Thus, mindfulness is hypothesized to facilitate processing efficiency (i.e., improve performance with no increase in effort). In contrast to mindfulness, TII are expected to impair performance effectiveness because they distract attention in the first stage. However, TRI are supposed to enhance the motivation to compensate for any impairment caused by TII; thus, TRI are hypothesized to predict an equal or greater level of task performance than can be expected with low TII. This effect can be achieved at the cost of diminished efficiency of recollection. In conclusion, TRI are assumed to improve effectiveness and diminish efficiency by increasing the effort put into the task performance.

\section{Event-Related Potential Components Related to Episodic Memory}

Dissociation of the processes involved in episodic memory can be made by means of electrophysiological measures, particularly the event-related potentials technique (ERP; for a review, see, e.g., Wilding \& Ranganath, 2012). In studies using ERP, two components associated with recognition memory have been commonly observed. The first, an early (300-500 ms from the stimulus onset) mid-frontal old/new effect has been linked with familiarity-based recognition (Curran, 2000; however, see Voss, Lucas, \& Paller, 2012, for a view that this effect reflects implicit priming rather than explicit familiarity). The second, a parietal late positive component (LPC), was observed in the 500-800 ms time window and has been associated with successful episodic retrieval, that is, recollection (Wilding \& Rugg, 1996). We expected that mindfulness correlates positively and TII correlate negatively with the LPC effect (at the first stage of recollection).

Although the mid-frontal old/new effect and LPC are the ERP components most commonly associated with retrieval processes, two late, post-retrieval potentials are also frequently reported in recognition studies. The right frontal old/new (RF) effect is usually observed in the 800-1500 ms time window (e.g., Hayama, Johnson, \& Rugg, 2008). Wilding and Ranganath (2012) proposed that this effect is related to generic monitoring processes activated when the outcome of retrieval is evaluated relative to a goal (e.g., selecting the proper response); it has also been suggested that the RF effect reflects uncertainty about retrieval result due to impoverished information (Hayama et al., 2008). The second post-retrieval component, the late posterior negativity (LPN), occurs in the same time window but has the opposite polarity and a posterior distribution (Johansson \& Mecklinger, 2003); it is thought to be involved in compensatory processes and may reflect a search for additional information, for example, retrieval of attribute conjunctions to support decisions about the source of retrieved information (Johansson \& Mecklinger, 2003). We assume that these two late components resemble information processing on the second stage of recollection. Thus, we expected that both RF and LPN effects would be positively predicted by the TRI (cf. Riby, Smallwood, \& Gunn, 2008) but not by mindfulness.

To sum up, we formulated the following hypotheses: (a) that state mindfulness would be negatively correlated to TII and TRI, (b) that mindfulness would predict an increase in performance effectiveness operationalized as an accuracy rate in the recollection task, (c) that mindfulness would be positively correlated to the LPC effect, and (d) that the opposite pattern of relationship between TII, performance effectiveness, and the LPC effect would occur. In other words, we expected that TII would negatively correlate with accuracy rate and strength of the LPC effect. Also, since TRI are supposed to enhance performance effectiveness at the cost of diminished processing efficiency, (e) we expected positive correlations between TRI and accuracy rate as well as the two compensatory ERP effects, RF and LPN.

\section{METHOD}

\section{Participants}

Thirty-one undergraduates ( 24 women, $M_{\text {age }}=22.8$ years, $S D=2.8$, range $=19-29)$ were recruited through advertisements at a local uni- 
versity; they received cinema vouchers in return for their participation. All participants had normal or corrected-to-normal vision (including normal color vision) and no history of neurological disorders. Data from two participants were excluded from the analysis because of very few artifact-free trials. All participants provided informed consent prior to the experiment and all procedures were approved by the Ethics Committee of the Institute of Psychology at the John Paul II Catholic University of Lublin.

\section{Materials}

\section{QUESTIONNAIRES}

To measure mindfulness during task performance we used five items drawn from the Mindfulness Attention Awareness Scale (MAAS; Brown \& Ryan, 2003), in line with suggestions of the authors, and modified them to capture awareness of the present state and a level of automaticity of task performance (e.g., "I did the task automatically, without being aware of what I was doing"). Participants rated each of the five items on a seven-point scale from 0 (not at all true for me) to 6 (very true for me); the item scores were reversed. The higher the total score, the higher the mindfulness during the task. In this study, the internal consistency for the MAAS state (measured as Cronbach's $\alpha$ ) was .64.

Two subscales of the Cognitive Interference Questionnaire (CIQ; Sarason \& Stoops, 1978) were used to measure a number of intrusive thoughts during the task. The Task-Related Interference subscale consists of 10 items that capture task-related intrusions (e.g., "I thought about how to work more carefully"), while mind-wandering was measured using the Task-Irrelevant Interference subscale, also consisting of 10 items (e.g., "I thought about other activities"). Participants responded by indicating how frequently each intrusive thought had occurred during the task, using a five-point scale from 1 (never) to 5 (very frequently). Both subscales had satisfactory reliability in this study ( $\alpha=$ .85 and $\alpha=.89$, respectively).

\section{RECOGNITION MEMORY TASK}

We used a recognition memory task similar to that used by Riby et al. (2008), which was modified from the procedure described by Curran (2000). Curran used concrete English nouns and manipulated plurality between practice and test trials to generate similar decoy items. We manipulated the color of the font in which the words were presented to differentiate between old and new but similar items. We used two colors, green and red. The stimuli were a set of 270 Polish concrete nouns varying from five to seven letters in length and from 50 to 99 occurrences per million words in frequency (Kazojć, 2011). The set of stimuli was divided into five lists: one 30-item list used in the practice block and four 60-item lists used in the four experimental blocks. Lists were matched for word length and frequency, and equal numbers of words were presented in each color in each block. All stimuli were presented in bold, 32-point Courier New font on a silver background at the center of a 17 in. LCD display with a resolution of $1024 \times 1028$ pixels and a refresh rate of $60 \mathrm{~Hz}$. Participants performed the task seated in a dimly lit, electrically shielded chamber, seated 50 $\mathrm{cm}$ from the computer monitor used to display the stimuli. The task was designed using E-Prime software.

\section{Procedure}

Participants completed the MAAS before being prepared for EEG recording and performing the recognition task. The procedure consisted of four study-test blocks ( 40 study trials and 60 test trials) separated by a buffer task. Each study trial comprised displaying the central fixation point $(500 \mathrm{~ms})$ followed by stimulus presentation $(1000 \mathrm{~ms})$, and then displaying a blank screen (1000 ms). Between the study and test phases, participants solved four multiple-choice (four responses) arithmetical problems (e.g., "90 - 64 + 76=?"). Each test trial began with displaying a fixation point $(500 \mathrm{~ms})$ followed by stimulus presentation. After 2.5 $s$ of displaying a word, a question mark appeared in the center of the screen with two response choices indicated below ("YES" and "NO"). Participants were instructed to answer yes if they had seen the stimulus during the preceding study phase or no if the stimulus had not been presented in the study phase or had been presented in a different color. Responses were indicated by pressing the appropriate button on the serial response box. Twenty out of 60 test stimuli were old (same word present in the same color as in the study phase), 20 were similar (presented in the study phase, but in a different color) and 20 were new items (not previously presented). The test trial ended when the participant pressed a button or after $2.5 \mathrm{~s}$ from the appearance of a question mark. Then the fixation point indicating the start of the next trial immediately appeared on the screen. The order in which the four practice-test blocks were presented and the order of stimuli within the blocks were randomized. Assignment of the stimuli to the three probe types (old, similar, and new) was counterbalanced across participants.

Immediately after finishing the recognition memory task, participants answered the questions (described above) from the MAAS and the CIQ, retrospectively assessing mindfulness and cognitive intrusions during the task. Participants were then debriefed. The whole experimental procedure lasted about $1.5 \mathrm{~h}$.

\section{EEG Recording and Analysis}

Continuous EEGs were recorded using a 64-channel Geodesic Sensor Net (Tucker, 1993) connected to an AC-coupled, high-input impedance amplifier (200 M $\Omega$, NetAmps, Electrical Geodesics, Inc.). Signals were recorded against the vertex $(\mathrm{Cz})$ electrode at a sampling rate of $250 \mathrm{~Hz}$ and were band-pass filtered (0.01-100 Hz). Impedance was maintained below $50 \mathrm{k} \Omega$, an acceptable range with this system, because of the high impedance of the amplifier. Offline, the signals were digitally filtered $(0.1-40 \mathrm{~Hz})$ and average-referenced.

Only EEG data from the test phases were analyzed. Artifacts were manually filtered for noticeable, nonstereotyped artifacts on the basis of careful visual inspection and then independent component analysis was used to reject well-characterized eye-blink, eye-movement, and muscle artifacts (Delorme, Sejnowski, \& Makeig, 2007). The EEG signals from each participant were first segmented into epochs beginning $200 \mathrm{~ms}$ prior to stimulus onset (word presentation in the 
recognition task) and ending $1.5 \mathrm{~s}$ after stimulus offset. Epochs were baseline-corrected relative to the mean amplitude during the prestimulus interval ( -200 to $0 \mathrm{~ms}$ ). Event-related potentials were computed for three response categories: correct recognition of old words, false recognition of similar words, and correct rejection of new words. The mean number of artifact-free trials in each of the three response categories was as follows: old $=44$ (range: 17-63); similar, $=27$ (range: 10-45); new, $=64$ (range: 43-79). Mean ERP amplitudes were computed for two consecutive time windows (500-800 and 800-1500 ms) to capture LPC, RF, and LPN effects. Detailed description of ERP analyses and results are presented in the supplemental material.

\section{RESULTS}

\section{Descriptive Statistics and Correlations Between Self-Report Measures}

Table 1 presents the ratio of correct responses for three stimulus types as averaged across participants. The new probes were easiest to respond to, with the accuracy rate nearing 0.9 . The old probes, compared to similar probes, were more difficult to recognize and accuracy rate for them was 0.09 above chance level.

Table 2 presents means, SDs, and correlations for the self-report measures. As expected, all measures that referred to a temporary state of mind during the task-state mindfulness, TII, and TRI-were moderately and significantly correlated with each other.

\section{Behavioral Measures of Effectiveness of Recollection Process}

To verify the hypothesis that mindfulness and cognitive intrusions are differently related to performance effectiveness, we used behavioral indices of recollection. Firstly, we tested a general linear mixed-effects

\begin{tabular}{lcc}
\hline \multicolumn{3}{l}{ TABLE 1. } \\
\multicolumn{3}{l}{ Average Proportions and SDs for Recognition Accuracy } \\
\hline Probe type & Hits & Misses \\
Old & $.59(.15)$ & $.41(.15)$ \\
\cline { 2 - 3 } Similar & Correct rejections & False alarms \\
New & $.64(.13)$ & $.36(.13)$ \\
\hline
\end{tabular}

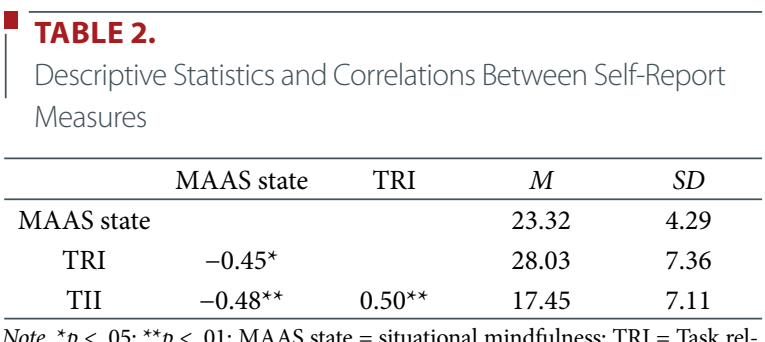

Note. ${ }^{\star} p<.05 ;{ }^{* *} p<.01$; MAAS state $=$ situational mindfulness; TRI $=$ Task relevant intrusions; TIT $=$ Task irrelevant intrusions. model for response accuracy in a particular trial $(0=$ incorrect, $1=$ correct) as the dependent variable. We chose multilevel modeling (MLM) to analyze our data because of several reasons. First, our data have a hierarchical structure, so MLM is a natural type of analysis for this kind (Nezlek, 2011). Second, MLM uses the full information present in data and allows for more precise estimation of SEs, which reduces the risk of the Type I error. Third, MLM used to analyze data from memory recognition tasks allows for obtaining results equivalent to signal detection theory (SDT) parameters and also solves some problems present in the traditional SDT approach (for a detailed explanation see Wright \& London, 2009) $)^{1}$.

All self-report measures (situational mindfulness, TII, and TRI) on Level 2 were grand-mean centered and then entered into the equation as predictors of the response accuracy in each of the trials (Level 1). We also included the interactions between probe type and each of the selfreport measures. Individual observations on Level 1 are cross-classified at Level 2 by both participants and stimuli, therefore, we entered intercepts independently for subjects and stimuli as random effects. To minimize the risk of Type I error, we also entered probe-type slopes as random effects varying across subjects. The $p$ values were adjusted with Tukey's method for comparing simple effects. All statistical analyses were performed using the $R$ program with the lme 4 package (Bates, Maechler, Bolker, \& Walker, 2015).

The whole model explained $25 \%$ of the variance of the accuracy rate (with $20 \%$ of the variance explained only by fixed effects; see Table $3)$. The analysis revealed a significant interaction between probe type and TRI, $\chi^{2}(2)=5.88, p<.05$. Situational mindfulness interacted with the probe type marginally, $\chi^{2}(2)=5.0, p=.08$, and TII had no effect on accuracy rate. To check whether the effect of TII disappears due to the mediation of mindfulness and/or TRI, we carried out an additional analysis with only TII and probe types as predictors and an interaction between them. We found no significant effects related to TII.

Although the interaction between situational mindfulness and the probe type was only marginally significant, we decided to deconstruct it because of its relevance to our hypothesis. The sign of simple effects suggested, as predicted, that the higher the situational mindfulness, the higher the probability of correct responses for old and new stimuli, $B=.19, S E=.14, p=.16$, and $B=.28, S E=.26, p=.27$, respectively; see Figure 1, Panel A). The level of situational mindfulness had no impact on the probability of correct responses for similar stimuli, $B=.04$, $S E=.14, p=.79$. Because all these effects are nonsignificant, they do not provide direct support for our hypothesis; however, marginal interaction effect between mindfulness and probe type suggests that mindfulness effects for old and new probes are slightly higher than for similar probes.

Analyses of simple effects showed also that TRI negatively and marginally predicted the probability of correct responses for new stimuli, $B=-.46, S E=.27, p<.09$, such that the higher the number of TRI, the lower the probability of correct rejections of new stimuli. However, in the case of old stimuli, the opposite, although nonsignificant effect was observed-the higher the number of TRI, the higher the probability of correct recognition of old stimuli, $B=.19, S E=.14, p=.18$; see Figure 
TABLE 3.

Fixed and Random Effects for the Model of Accuracy Rate

\begin{tabular}{lcccll}
\hline Fixed effects & $\chi^{2}$ & $d f$ & $p$ & Random effects & \\
\hline (Intercept) & 144.8 & 1 & .001 & $\sigma 2$ & 3.29 \\
MAAS state & 1.21 & 1 & .27 & $\tau_{\text {oo } \text { Word }}$ & 0.05 \\
TII & 0.37 & 1 & .54 & $\tau_{\text {oo Subject }}$ & 1.17 \\
TRI & 2.93 & 1 & .09 & $\tau 11_{\text {Subjectsimilar }}$ & 0.27 \\
Probe type & 247.5 & 2 & .001 & $\tau_{11}$ subjecttarget & 1.63 \\
MAAS state $\times$ probe type & 5.0 & 2 & .08 & ICC $_{\text {Word }}$ & 0.01 \\
TII $\times$ probe type & 1.4 & 2 & .50 & ICC $_{\text {Subject }}$ & 0.26 \\
TRI $\times$ probe type & 5.88 & 2 & .05 & Observations & 6925 \\
& & & & Marginal $\mathrm{R}^{2} /$ Conditional $\mathrm{R}^{2}$ & $0.20 / 0.25$ \\
\hline
\end{tabular}

Note . MAAS state = situational mindfulness; TRI = Task relevant intrusions; TII = Task irrelevant intrusions

1, Panel B). Task-relevant intrusions had no effect on the recognition of similar stimuli, $B=-.05, S E=.14, p=.72$. However, we observed significant difference between TRI slopes for new versus similar probes $(\Delta B=-.41, S E=.17, p<.05)$ and marginal difference between TRI slopes for new versus old probes $(\Delta B=-.65, S E=.31, p=.097)$. These results suggest that TRI are related to the accuracy rate in new versus old and similar probes in opposite direction.

\section{Modulation of Event-Related Potential Components by Mindfulness and Cognitive Intrusions}

Means and SDs for ERP amplitudes for each of the probe types are presented in Table 4. To verify the hypothesis that mindfulness and cognitive intrusions are related to ERP components, we tested linear mixed-effects models with the amplitudes of three ERP components (LPC, RF, and LPN) averaged for each probe type (correct recognition of old stimuli, correct rejection of new stimuli, and false recognition of similar stimuli) as dependent variables. With each of the models, we allowed the intercept to vary across participants (random effect). Parameters in the models were computed with the maximum likelihood estimator. We entered situational mindfulness, TII, and TRI into

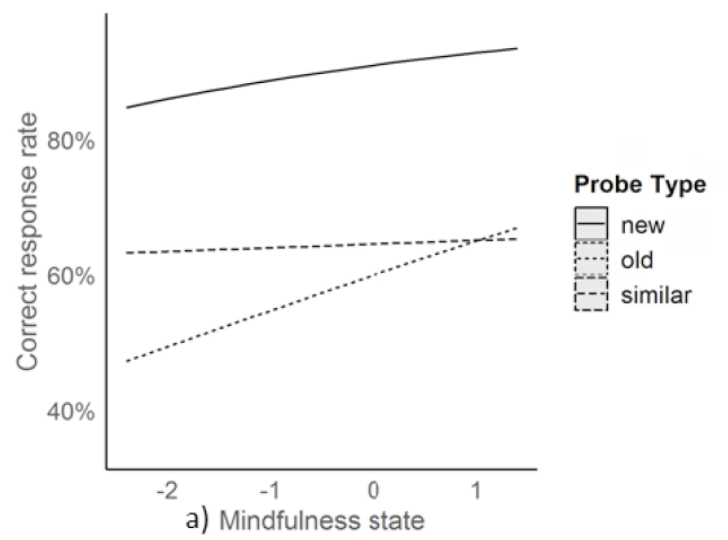

the models, as well as their interactions with probe type as fixed effects (see Table 5). The p values were adjusted with Tukey's method for comparing simple effects.

\section{LATE PARIETAL COMPONENT AMPLITUDE}

The whole model explained $80 \%$ of the LPC amplitude variance, with $16 \%$ assigned only to fixed effects. There was only one significant effect-a main effect of probe type, $\chi^{2}(2)=21.9, p<.001$. We also observed a marginal effect of mindfulness, $\chi^{2}(1)=3.68, p<.06$. It means that the higher the mindfulness during the task, the lower the amplitude of ERP regardless of the probe type. No other significant interaction effects were found.

\section{RIGHT FRONTAL AMPLITUDE}

The whole model explained 59\% of the variability in RF amplitude, with fixed effects explaining 32\%. A significant interaction between probe type and TRI was observed, $\chi^{2}(2)=10.46, p<.005$ : TRI positively predicted RF amplitude only for correctly recognized old probes, $B=.76, S E=.21, p<.001$, whereas for the correctly rejected new and incorrectly recognized similar probes, the effect of TRI was negligible, $B=.009, S E=.21, p=.97$ and $B=.33, S E=.21, p=.13$, respectively. In other words, these results suggest that TRI are positively related to the

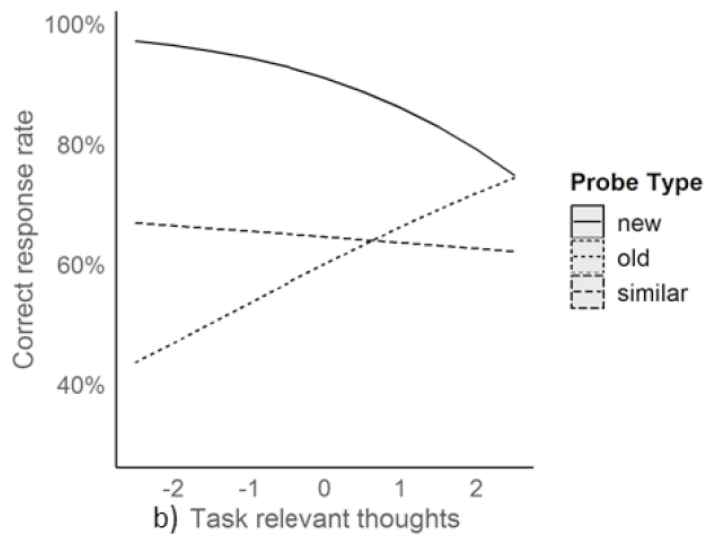

|FIGURE 1.

Effect of mindfulness state (Panel A) and task relevant intrusions (TRl; Panel B) on correct response rate depending on the probe type. Mindfulness and TRI scales are in SDs. 

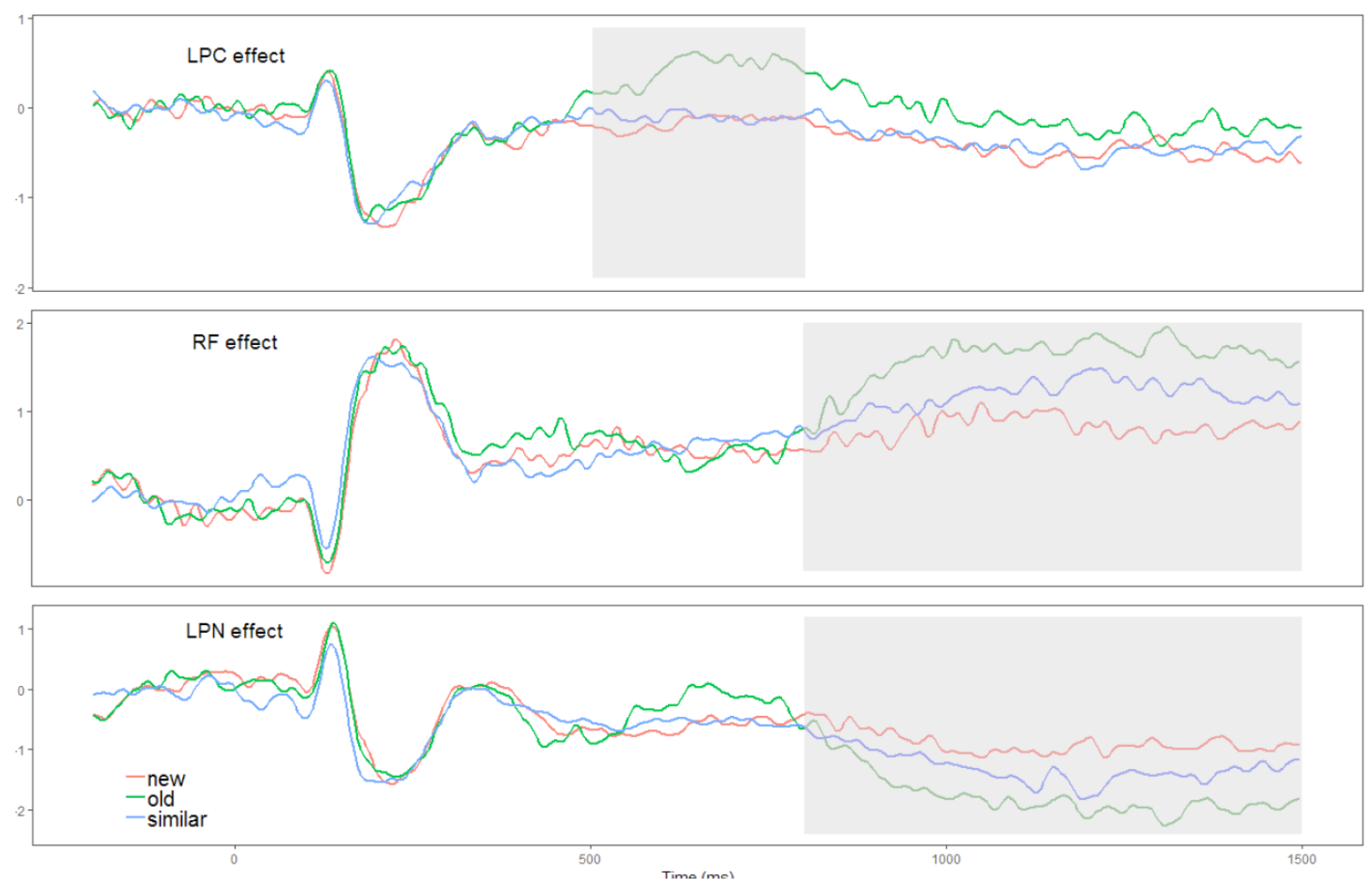

FIGURE 2.

Grand-averaged ERPs from the recognition task for main electrodes in left-parietal (top), right-frontal (middle) and mid-parietal (bottom) regions. Grey rectangles indicate time windows with observed significant differences in amplitudes between probe types.

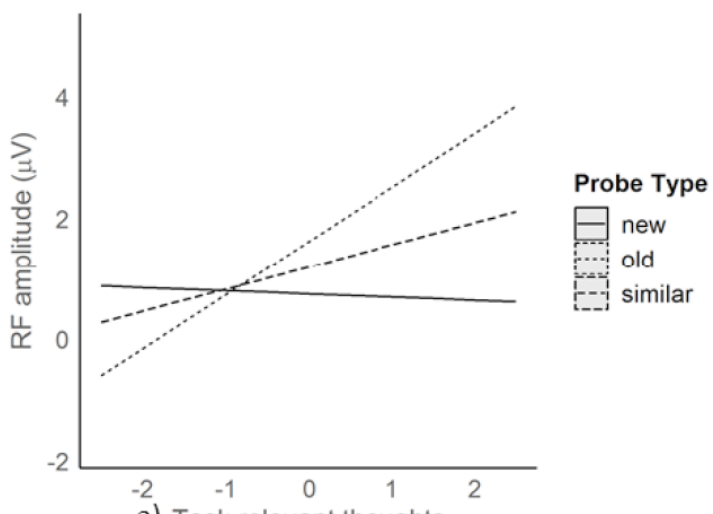

a) Task relevant thoughts

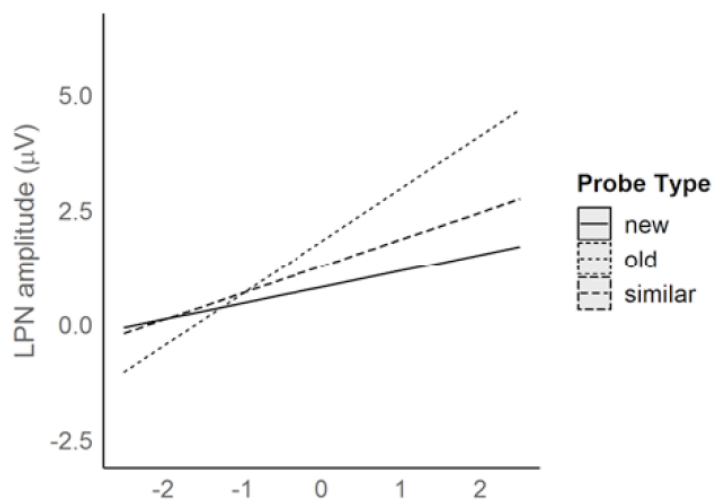

b) Task relevant thoughts

FIGURE 3.

Effects of task related intrusions on average amplitude of probe types regarding right frontal (RF, Panel A) and late posterior negativity (LPN, Panel B) components. Task-relevant intrusion scale is in SDs.

strength of the RF effect (see Figure 3, Panel A). No significant effects related to mindfulness were observed.

\section{LATE POSTERIOR NEGATIVITY AMPLITUDE}

The model explained $67 \%$ of LPN amplitude variability (30\% was explained by fixed effects). We observed one significant interaction between probe type and TRI, $\chi^{2}(2)=6.82, p<.03$. Task-relevant in- trusions predicted LPN amplitude for old probes, $B=1.18, S E=.29$, $p<.001$, and for similar probes, $B=.71, S E=.29, p<.05$, but not for new probes, $B=.45, S E=.29, p=.13$. This suggests that TRI are also positively associated with the strength of the LPN effect (see Figure 3, Panel B). No other effects were observed. 


\section{TABLE 4.}

Means and SDs for Event-Related Potential Amplitudes Averaged Across Probe Types

\begin{tabular}{lcccccc}
\hline \multirow{2}{*}{ Probe type } & \multicolumn{2}{c}{ LPC } & \multicolumn{2}{c}{ RF } & \multicolumn{2}{c}{ LPN } \\
\cline { 2 - 7 } & $M$ & $S D$ & $M$ & $S D$ & $M$ & $S D$ \\
\cline { 2 - 7 } New & -0.16 & 0.99 & 0.82 & 0.94 & -0.89 & 1.53 \\
Similar & -0.1 & 1.09 & 1.17 & 0.88 & -1.29 & 1.39 \\
Old & 0.43 & 1.53 & 1.6 & 1.59 & -1.75 & 2 \\
\hline
\end{tabular}

Note LPC = late parietal component; RF = right frontal component; $\mathrm{LPN}=$ late posterior negativity.

\section{DISCUSSION}

The main aim of the present study was to test several hypotheses concerning the relationships between mindfulness, cognitive intrusions, performance effectiveness, and processing efficiency. As expected, all self-report measures regarding mindfulness during task performance as well as TII and TRI were significantly correlated. Mindfulness, that is, acting with awareness, predicted both TII and TRI. However, we cannot equate these phenomena because of a moderate magnitude of the correlations. These simple results confirm the claim of Brown et al. (2011) that mindfulness cannot be reduced to attentional control (see also Jankowski \& Bąk, 2019). The question is, however, whether mindfulness and cognitive intrusions play different roles in the effectiveness and efficiency of task performance, as we hypothesized.

Participants with high, as opposed to low task-related mindfulness had a slightly higher accuracy rate for old and new stimuli than participants with low task-related mindfulness. This effect was weak and nonsignificant. Therefore, although it was in line with our expectation, it did not constitute a definite confirmation of the hypothesis. However, because we observed a difference between mindfulness effects for similar versus old and new probes, the results suggest that mindfulness predicts success rather in recognition than in recollection. Moreover, we did not find evidence from ERP amplitude analyses that mindfulness improves the LPC effect associated with retrieving information in the first stage of recollection. In fact, there was an overall, negative and marginal effect of mindfulness on LPC amplitudes in all types of probes. This might indicate that mindfulness is not related to recollection (which is indexed by LPC) and that it does not enhance source memory, but rather predicts better recognition memory performance on the basis of familiarity, an automatic and nonspecific feeling of memory that can also support retrieval. This is consistent with a recent work by Rosenstreich and Ruderman (2017), who, in two studies, found that mindfulness was associated with familiaritybased "know" responses in the remember/know procedure (Study 2) and that one facet of mindfulness, i.e. observing, predicted rates of familiarity (Study 3). Also, our behavioral data speak in favor of this interpretation, as mindfulness allowed for slightly higher accuracy for hits and new probes (which can be guided by familiarity or lack of familiarity, respectively), but not for similar probes (which can be more recollection-based as rejecting a similar item might require recollecting details-font color-associated with it).

We found no evidence that TII predicts performance effectiveness. The effect of TII is not also explained by mindfulness and/or TRI. In our study, mind-wandering did not distract participants enough to significantly diminish their performance. Although the hypothesis was not confirmed, this result is not surprising. Because the task presented in the study was difficult, mind-wandering was probably reduced. This effect has been observed in earlier studies (e.g., Teasdale et al., 1995). We suppose that TII could affect performance more in easier tasks (e.g., with fewer items to remember in a single block). In difficult tasks, we might expect intensifying TRI instead of TII, which was the case in our study.

TABLE 5.

Fixed and Random Effects for models of Event-Related Potential Components Associated With Recollection

\begin{tabular}{lcccccc}
\hline \multirow{2}{*}{ Fixed effects } & \multicolumn{2}{c}{ LPC } & \multicolumn{2}{c}{ RF } & \multicolumn{2}{c}{ LPN } \\
\cline { 2 - 7 } & $\chi^{2}$ & $p$ & $\chi^{2}$ & $p$ & $\chi^{2}$ & $p$ \\
\hline MAAS state & 3.68 & 0.06 & 1.46 & 0.23 & 0.70 & 0.40 \\
TII & 0.31 & 0.58 & 0.76 & 0.38 & 0.008 & 0.93 \\
TRI & 0.69 & 0.41 & 0.002 & 0.97 & 2.39 & 0.12 \\
Probe type & 21.9 & 0.001 & 20.67 & 0.001 & 18.73 & 0.001 \\
MAAS state $\times$ probe type & 0.39 & 0.82 & 0.32 & 0.85 & 4.38 & 0.11 \\
TII $\times$ probe type & 2.44 & 0.30 & 3.43 & 0.18 & 0.63 & 0.73 \\
TRI $\times$ probe type & 0.75 & 0.69 & 10.46 & 0.005 & 6.82 & 0.03 \\
\hline Random effects & & & & & \\
$\sigma 2$ & 0.34 & 0.54 & 0.79 \\
$\tau_{\text {oo Subject }}$ & 1.05 & 0.37 & 0.91 \\
ICC ${ }_{\text {Subject }}$ & 0.76 & 0.41 & 0.54 \\
Observations & \multicolumn{2}{c}{87} & 87 & 87 \\
Marginal R $^{2} /$ Conditional R & $0.16 / 0.80$ & $0.32 / 0.59$ & $0.30 / 0.67$
\end{tabular}

Note MAAS state $=$ situational mindfulness; TRI $=$ Task relevant intrusions; TII $=$ Task irrelevant intrusions; $\mathrm{LPC}=$ late parietal component; $\mathrm{RF}=$ right frontal component; $\mathrm{LPN}=$ late posterior negativity. 
The pattern of relationships between TRI and ERP components confirmed another hypothesis: TRI positively predicted the strength of both RF and LPN effects. In other words, the more task-related worrying, the more effort is put into correct recollection, as manifested in RF and LPN effects. In this aspect, our results were similar to those found by Riby et al. (2008).

Although TRI facilitate compensatory processes, our results reveal a complex picture of the relationship between TRI and quality of performance. We observed different effects of TRI on the accuracy rate for old and new probes: While TRI were correlated with an increase in accuracy for old probes, they also predicted a decrease in the probability of correct rejections of new probes. We propose that the compensatory processes associated with TRI have opposite effects in responding to new versus old probes. Correct rejections of new probes are relatively easy and can be made automatically. This effect is related to the fact that for new probes, no information retrieved from memory needs to be evaluated because, by definition, there is no information regarding the new probes encoded in the practice stage. A simple rejection in the case of a lack of signal about the previous occurrence of a stimulus is the best response. However, compensatory strategies involving enhanced monitoring of contextual information can introduce some hesitation in responding to each kind of probe. While this is beneficial for old probes because they could be evaluated more precisely, for new probes it is detrimental because automatic responding is inhibited, which can cause errors in some cases. Although an alternative explanation is also possible in light of the SDT (in terms of change in response bias; Miller \& Wolford, 1999) we agree with Roediger and McDermott (1999) that this is rather improbable because of a lack of change in accuracy rate for similar probes.

The results suggest that neither mindfulness nor TRI predicted accuracy for similar probes. We think that this result may be understood if we take a more complex perspective on the processes involved in the recognition task. While most researchers are interested only in the manifestation of explicit memory (i.e., processes associated with familiarity and recollection effects that can be consciously reported in the "know/remember" procedure), some of them claim that implicit processes should also be taken into consideration (e.g., Voss et al., 2012). The vast literature on the high rate of false alarms in the case of words similar to the target stimuli shows that the effect of implicit memory (i.e., semantic priming) is very strong and irresistible even if participants are informed about it and warned to be mindful (Gallo, Roberts, \& Seamon, 1997). If our interpretation is true, mere state of mindfulness is not sufficient to protect against false memories based on implicit memory associated with similar probes (for comparable results, see also Rosenstreich, 2016, and Wilson et al., 2015). However, because several studies found that in specific conditions, mindfulness reduces false memories (e.g., when induced after the encoding stage, Calvillo, Flores, \& Gonzales., 2018), further research is needed to explain these inconsistencies. The question remains whether other facets of mindfulness, such as nonreactiveness (conceptualized as a state), could be better predictors of accuracy in the case of similar probes.
Two main issues limit the generalizability of the presented results. The first refers to the small sample we used in our study. Although small samples are frequently used in EEG studies, because we correlated ERP effects with self-report measures, our findings can be treated as preliminary and need replication in further research. The second issue relates to the design of our study. We found theoretically sound relationships between core variables, but we cannot determine whether mindfulness indeed influences recognition or whether possessing a good memory, and-in a broader view-efficient executive functions, improves the ability to be mindful. Future experimental research should investigate this problem directly.

Despite these limitations, important conclusions can be derived from our study. First, we replicated all three ERP effects associated with the recollection process, which proves the validity of the study design. Second, we found a marginal but expected effect suggesting that state mindfulness, that is, acting with open awareness, predicts better performance in the recognition task. This result partly confirms the predictions from the model by Levi and Rosenstreich (2018). Moreover, mindfulness-related improvement was not associated with the higher effort due to compensatory processes. We also observed a significant relationship between TRI and late ERP effects, which directly confirms the attentional control theory (Eysenck et al., 2007): worrying about task performance seems to trigger compensatory processes, as this theory predicts. The results suggest that mindfulness, understood as acting with awareness, contributes to item recognition independently from mind-wandering and differently from worrying, which plays a motivational role. The effect of TRI is ambivalent, however. The results suggest that increased TRI shift the focus of attention from detection of the familiarity effect to contextual information; this results in better recognition of old stimuli and a higher rate of false recognitions of new stimuli. Further research is needed to examine the role of other facets of mindfulness, for example, a state of nonreactiveness, in better inhibition of false recalls of lures.

\section{ACKNOWLEDGEMENTS}

We would like to thanks to Piotr Francuz, Paweł Augustynowicz, Andrzej Cudo, Agnieszka Fudala-Czyż, Agnieszka Krzysztofiak, Łukasz Nikel, Przemysław Tużnik, Emilia Zabielska-Mendyk, and Dariusz Zapała for their help in conducting the study.

\section{FOOTNOTES}

${ }^{1}$ Correlations between mindfulness, task-irrelevant intrusions, task-relevant intrusions, and signal detection theory parameters as well as regression analyses with overall sensitivity and bias parameters as dependent variables are presented in the supplemental material.

\section{REFERENCES}

Alberts, H. J., Otgaar, H., \& Kalagi, J. (2017). Minding the source: The impact of mindfulness on source monitoring. Legal and Criminological Psychology, 22, 302-313. doi: 10.1111/ Icrp.12102 إلسلس

Alberts, H. J., \& Thewissen, R. (2011). The effect of a brief mind- 
fulness intervention on memory for positively and negatively valenced stimuli. Mindfulness, 2, 73-77. doi:10.1007/s12671011-0044-7 سلس سلس

Bates, D., Maechler, M., Bolker, B., \& Walker, S. (2015). Fitting linear mixed-effects models using Ime4. Journal of Statistical Software, 67, 1-48. السلسلس

Brown, K. W., Goodman, R. J., Ryan, R. M., \& Anālayo, B. (2016). Mindfulness enhances episodic memory performance: Evidence from a multimethod investigation. PLoS One, 11, e0153309. doi:10.1371/journal.pone.0153309 السلسلس

Brown, K. W., \& Ryan, R. M. (2003). The benefits of being present: Mindfulness and its role in psychological well-being. Journal of

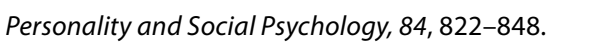

Brown, K. W., Ryan, R. M., Loverich, T. M., Biegel, G. M., \& West, A. M. (2011). Out of the armchair and into the streets: Measuring mindfulness advances knowledge and improves interventions: Reply to Grossman (2011). Psychological Assessment, 23, 1041-1046. doi:10.1037/a0025781 16

Calvillo, D. P., Flores, A. N., \& Gonzales, L. C. (2018). A brief mindfulness induction after encoding decreases false recognition in the Deese-Roediger-McDermott paradigm. Psychology of Consciousness: Theory, Research, and Practice. Advance online publication. doi:10.1037/cns0000145 السلسلس

Curran, T. (2000). Brain potentials of recollection and familiarity. Memory \& Cognition, 28, 923-938. doi:10.3758/BF03209340 السلسلسلس

Delorme, A., Sejnowski, T., Makeig, S. (2007). Enhanced detection of artifacts in EEG data using higher-order statistics and independent component analysis. Neurolmage, 34, 1443-1449. doi:10.1016/j.neuroimage.2006.11.004 سلس

Elward, R. L., Evans, L. H., \& Wilding, E. L. (2013). The role of working memory capacity in the control of recollection. Cortex, 49 ,

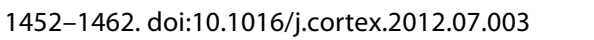

Eysenck, M. W., Derakshan, N., Santos, R., \& Calvo, M. G. (2007). Anxiety and cognitive performance: Attentional control theory. Emotion, 7, 336-353. doi:10.1037/1528-3542.7.2.336 السلسلس

Eysenck, M. W., \& Derakshan, N. (2011). New perspectives in attentional control theory. Personality and Individual Differences, 50, 955-960. doi:10.1016/j.paid.2010.08.019 المالسلسلة

Gallo, D. A., Roberts, M. J., \& Seamon, J. G. (1997). Remembering words not presented in lists: Can we avoid creating false memories? Psychonomic Bulletin \& Review, 4, 271-276. doi:10.3758/ BF03209405 السلسلس

Grossman, P. (2011). Defining mindfulness by how poorly I think I pay attention during everyday awareness and other intractable problems for psychology's (re)invention of mindfulness: Comment on Brown et al. (2011). Psychological Assessment, 23, 1034-1040. doi:10.1037/a0022713 友

Hayama, H. R., Johnson, J. D., Rugg, M. D. (2008). The relationship between the right frontal old/new ERP effect and post-retrieval monitoring: Specific or nonspecific? Neuropsychologia, 46, 1211-1223. doi:10.1016/j.neuropsychologia.2007.11.021 المالسلسل Hayes, S., Hirsch, C.R., \& Mathews, A., (2008). Restriction of working memory capacity during worry. Journal of Abnormal Psychology, 117, 712-717. doi:10.1037/a0012908 السلسلس

Heeren, A., Van Broeck, N., \& Philippot, P. (2009). The effects of mindfulness on executive processes and autobiographical memory specificity. Behaviour Research and Therapy, 47, 403-

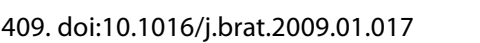

Jankowski, T., \& Bąk, W. (2019). Mindfulness as a mediator of the relationship between trait anxiety, attentional control and cognitive failures. A multimodel inference approach. Personality and Individual Differences, 142, 62-71. doi:10.1016/j. paid.2019.01.034 السلسلس

Jankowski, T. \& Holas, P. (2014). Metacognitive model of mindfulness. Consciousness and Cognition, 28, 64-80. Doi:10.1016/j. concog.2014.06.005 البلسلس

Jha, A. P., Krompinger, J., \& Baime, M. J. (2007). Mindfulness training modifies subsystems of attention. Cognitive, Affective \& Behavioral Neuroscience, 7, 109-119. doi:10.3758/CABN.7.2.109 |لسلس

Johansson, M., Mecklinger, A. (2003). The late posterior negativity in ERP studies of episodic memory: Action monitoring and retrieval of attribute conjunctions. Biological Psychology, 64, 91-117. doi:10.1016/S0301-0511(03)00104-2 سلس سلس

Kabat-Zinn, J. (2003). Mindfulness-based interventions in context: Past, present, and future. Clinical Psychology: Science and Practice, 10, 144-156. doi:10.1093/clipsy.bpg016 السلسلس

Kane, M. J., Brown, L. H., McVay, J. C., Silvia, P. J., Myin-Germeys, I., \& Kwapil, T. R. (2007). For whom the mind wanders, and when: An experience-sampling study of working memory and executive control in daily life. Psychological Science, 18, 614-621. doi:10.1111/j.1467-9280.2007.01948.x سلس

Kazojć, J. (2011). Słownik frekwencyjny języka polskiego [The frequency dictionary of the Polish language]. Retrieved from: http://www.slowniki.org.pl/i27.html

Knott, L. M., \& Dewhurst, S. A. (2007). The effects of divided attention at study and test on false recognition: A comparison of DRM and categorized lists. Memory and Cognition, 35, 1954-1965. doi:10.3758/BF03192928 سلس

Levi, U. \& Rosenstreich, E. J. (2018). Mindfulness and memory: A review of findings and a potential model. Journal of Cognitive Enhancement. Retrieved from: https://link.springer.com/article/10.1007/s41465-018-0099-7

Lloyd, M., Szani, A., Rubenstein, K., Colgary, C., \& Pereira-Pasarin, L. (2016). A brief mindfulness exercise before retrieval reduces recognition memory false alarms. Mindfulness, 7, 606-613. doi: 10.1007/s12671-016-0495-y سلس

Maillet, D. \& Rajah, M. N. (2013). Age-related changes in frequency of mind-wandering and task-related interferences during memory encoding and their impact on retrieval. Memory, 21, 818-831. doi:10.1080/09658211.2012.761714 البلسلس

Miller, M. B., \& Wolford, G. L. (1999). Theoretical commentary: The role of criterion shift in false memory. Psychological Review, 106, 398-405. doi:10.1037/0033-295X.106.2.398 السلسلس 
NetAmps [Apparatus]. Eugene, OR: Electrical Geodesics, Inc. Nezlek, J. B. (2011). The SAGE library of methods in social and personality psychology. Multilevel modeling for social and personality psychology. Thousand Oaks, CA: Sage Publications.

Norris, C. J., Creem, D., Hendler, R., \& Kober, H. (2018). Brief mindfulness meditation improves attention in novices: Evidence from ERPs and moderation by neuroticism. Frontiers in Human Neuroscience, 12, 315. doi:10.3389/fnhum.2018.00315 السلسلس

Riby, L. M., Smallwood, J., \& Gunn, V. P. (2008). Mind wandering and retrieval from episodic memory: A pilot event-related potential study. Psychological Reports, 102, 805-818. doi:10.2466/ pr0.102.3.805-818 إلسلس

Roediger, H. L. III, \& McDermott, K. B. (1999). False alarms and false memories. Psychological Review, 106, 406-410. doi:10.1037/0033-295X.106.2.406 المالسلسلسا Rest

Rosenstreich, E. (2016). Mindfulness and false-memories: The impact of mindfulness practice on the DRM paradigm. The Journal of Psychology: Interdisciplinary and Applied, 150, 58-71. doi:10.1080/00223980.2015.1004298 السلسلس

Rosenstreich, E., Goshen-Gottstein, Y. (2015). Recollection-based retrieval Is influenced by contextual variation at encoding but not at retrieval. PLoS One, 10, e0130403. doi:10.1371/journal. pone.0130403 سلس

Rosenstreich, E., \& Ruderman, L. (2016). Not sensitive, yet less biased: A signal detection theory perspective on mindfulness, attention, and recognition memory. Consciousness and Cognition, 43, 48-56. doi:10.1016/j.concog.2016.05.007 السلسلسلس

Rosenstreich, E., \& Ruderman, L. (2017). A dual-process perspective on mindfulness, memory, and consciousness. Mindfulness, 8, 505-516. doi:10.1007/s12671-016-0627-4 سلس الس

Sarason, I. G., Sarason, B. R., Keefe, D. E., Hayes, B. E., \& Shearin, E. N. (1986). Cognitive interference: Situational determinants and traitlike characteristics. Journal of Personality and Social Psychology, 51, 215-226. doi:10.1037/0022-3514.51.1.215 البلسلس

Sarason, I. G., \& Stoops, R. (1978). Test anxiety and the passage of time. Journal of Consulting and Clinical Psychology, 46, 102-109.

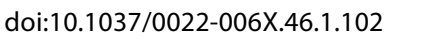

Smallwood, J., Davies, J. B., Heim, D., Finnigan, F., Sudberry, M., O'Connor, R. \& Obonsawin, M. (2004). Subjective experience and the attentional lapse: Task engagement and disengagement during sustained attention. Consciousness and Cognition,
13, 657-690. doi:10.1016/j.concog.2004.06.003 |لإلس

Teasdale, J. D., Dritschel, B. H., Taylor, M. J., Proctor, L., Lloyd, C. A., Nimmo-Smith, I., \& Baddeley, A. (1995). Stimulus-independent thought depends on central executive resources. Memory \& Cognition, 23, 551-559. doi:10.3758/BF03197257 السلسلس

Thomson, D. R., Smilek, D., Besner, D. (2014). On the asymmetric effects of mind-wandering on levels of processing at encoding and retrieval. Psychonomic Bulletin \& Review, 21, 728-733. doi:10.3758/s13423-013-0526-9 المالسلس

Tucker, D. M. (1993). Spatial sampling of head electrical fields: The geodesic sensor net. Electroencephalography \& Clinical Neurophysiology, 87, 154-163. doi:10.1016/00134694(93)90121-B الم السلسلس

van Vugt, M. K. (2015). Cognitive benefits of mindfulness meditation. In K. W. Brown, J. D. Creswell, \& R. M. Ryan (Eds.), Handbook of mindfulness: Theory, research, and practice (pp. 190-207). New York City, NY: Guilford Press.

Voss, J. L., Lucas, H. D., \& Paller, K. A. (2012). More than a feeling: Pervasive influences of memory processing without awareness of remembering. Cognitive Neuroscience, 3, 193-207. do i:10.1080/17588928.2012.674935 إلس

Wilding, E. L. and Ranganath, C. (2012). Electrophysiological correlates of episodic memory processes. In: Luck, S. and Kappenman, E. (eds.), The Oxford Handbook of Event-Related Potential Components (pp. 373-396). Oxford, England: Oxford University Press.

Wilding, E. L., Rugg, M. D. (1996). An event-related potential study of recognition memory with and without retrieval of source. Brain, 119, 889-905. doi:10.1093/brain/119.3.889 السلسلس

Wilson, B. M., Mickes, L., Stolarz-Fantino, S., Evrard, M., \& Fantino, E. (2015). Increased false-memory susceptibility after mindfulness meditation. Psychological Science, 26, 1567-1573. doi:10.1177/0956797615593705 الف السلس

Wright, D. B., \&London, K. (2009). Multilevel modelling: Beyond the basic applications. British Journal of Mathematical and Statistical

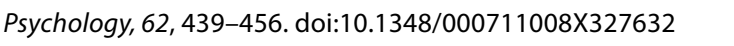
Yonelinas, A. P. (2002). The nature of recollection and familiarity: A review of 30 years of research. Journal of Memory and

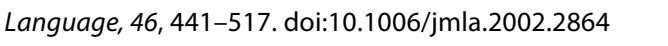

RECEIVED 01.02.2019| ACCEPTED 05.04.2019 